\title{
2D Hall Sensor Array for Measuring the Position of a Magnet Matrix
}

\author{
Hyeong Joon Ahn ${ }^{1, \#}$ and Kyoung Rock Kim² \\ 1 Department of Mechanical Engineering, Soogsil Unviersity, Seoul, South Korea \\ 2 Graduate School. Department of Mechanical Engineering, Soogsil Unviersity, Seoul, South Korea \\ \# Corresponding Author / E-mail: ahj123@ssu.ac.kr, TEL: +82-2-820-0654, FAX: +82-2-8200668
}

KEYWORDS: Hall sensor, Sensor array, Filter, Harmonic components, Displacement sensor for magnet matrix.

\begin{abstract}
Hall sensor array is used to measure the position of a magnet array and needs compensation of errors caused by manufacturing tolerances as well as harmonic components of the magnetic flux of the magnet array. This paper presents a $2 D$ hall sensor array to measure the position of a magnet matrix by filtering harmonic components. First, $1 D$ sensor array to filter out arbitrary number of harmonic signals is proposed and extended to $2 D$ sensor array for the position measurement of a magnet matrix. A 2D hall sensor array for filtering both fundamental and $2^{\text {nd }}$ harmonic components of the magnetic flux and its conditioning circuit board are built to measure the position of the magnet matrix for a planar motor. Finally, performances of filtering harmonics and measuring position of the magnet matrix are experimentally verified with a XY linear motion stage. The proposed $2 D$ hall sensor array is compatible to a sine encoder and is directly applicable to commercial motion controllers.
\end{abstract}

\section{NOMENCLATURE}

$A=$ Sensor gain

$f=$ Sensor output

$L_{i}=\mathrm{i}^{\text {th }}$ Sensor location

$W_{n}=$ Total width of sensor array of $n$ sensors

$x=$ Physical quantity (displacement)

$\alpha=$ Phase angle of harmonic component

$\lambda=$ Wavelength of harmonic component

\section{Introduction}

Manufacturing equipment usually needs precise multi-degree of freedom (DOF) motions as well as compact, lightweight and high-stiff structures. For the multi-DOF motion stage, conventional stacked, gantry and hybrid structures have been used so far. However, these conventional structures are reaching the limit to satisfy pressing demands for fast-response and high-precision motion. ${ }^{1}$

Planar motors have been studied in several research groups to meet the high-speed, precise, compact and lightweight needs for the multi-
DOF motion stage..$^{2-9}$ Although there are several types such as moving coil type ${ }^{4}$ and reluctant motor, ${ }^{10}$ planar motors with moving magnet matrix were mostly studied. , $3,5-9^{-1}$

There are several sensing options for planar motors such as interferometry, ${ }^{2,5}$ capacitance gauge, ${ }^{2}$ encoder, ${ }^{11,12}$ hall sensors ${ }^{13,14}$ and vision sensor. ${ }^{15}$ Using hall sensors is most cost-effective methodology since the magnet matrix is used as the measured target and various hall sensors are available at low cost. However, magnetic flux density distribution of the magnet matrix does not have perfect sinusoidal wave due to harmonics and manufacturing tolerance. Therefore, error separation technique is required to improve the accuracy. ${ }^{14}$ In addition, the hall sensors need customized interface rather than standard interface of general motion controller.

Harmonic components of magnetic flux distribution of the magnet matrix can hardly be removed using conventional filter techniques since frequencies of harmonic components vary according to the motion speed of the mover. Halbach magnet array can significantly reduce harmonic components of magnetic flux density. ${ }^{14,16}$ In addition, periodic (harmonic) signals can be separated using multi-sensors (or sensor array). ${ }^{17-19}$ If the harmonic components are removed, we can save considerable efforts on error compensation and improve intrinsic accuracy of the sensing system. 
In this paper, we propose a $2 \mathrm{D}$ hall sensor array to measure the position of a magnet matrix by filtering harmonic components of magnetic flux of the magnet matrix. First, 1D sensor array to filter out arbitrary number of harmonic signals is proposed and extended to a $2 \mathrm{D}$ sensor array for position measurement of a magnet matrix. A 2D hall sensor array and its conditioning circuit are built to measure the position of the magnet matrix for a planar motor. The $2 \mathrm{D}$ hall sensor array is directly applicable to commercial motion controller because of its compatibility to a typical sine encoder. Finally, the harmonic filtering performance of the 2D hall sensor array is experimentally verified with a XY linear motion stage.

\section{Sensor Array for Filtering of Harmonic Signals}

\subsection{D Sensor Array}

At least, two sensors $\left(s_{1}\right.$ and $s_{2}$ ) are required to remove a harmonic component of wave length $\lambda_{1}$. Two sensors should be separated in half wavelength (or $\pi$ phase difference) of the harmonic component. ${ }^{14}$ That is, a sensor has output of $f\left(x \lambda_{1} / 4\right)$ and the other sensor has output of $f\left(x+\lambda_{1} / 4\right)$, where $x$ is a dependent variable for a physical quantity (displacement). If the sensor output $f$ is a pure harmonic function of $A_{1} \sin \left(2 \pi x / \lambda_{1}\right)$, sum of two sensor outputs always becomes zero, as shown in Eq. (1) and Fig. 1(a).

$$
A_{1} \sin (\pi x / \lambda-\pi / 2)+A_{1} \sin \pi x / \lambda_{1}+(\pi / 2)=0
$$

If we want to remove another harmonic component of wavelength $\lambda_{2}$ at the same time, we have to divide one sensor $\left(s_{1}\right.$ or $\left.s_{2}\right)$ into two sensors $\left(s_{1}^{\prime}\right.$ and $s_{2}^{\prime}$ or $s_{3}^{\prime}$ and $\left.s_{4}^{\prime}\right)$. In addition, the locations of the sensors should be separated in half wavelength of $\lambda_{2}$ as shown in Fig. 1(b). Then, four sensor outputs should be $f\left(x \lambda_{1} / 4 \lambda_{2} / 4\right), f\left(x \lambda_{1} / 4+\lambda_{2} / 4\right)$, $f\left(x+\lambda_{1} / 4 \lambda_{2} / 4\right)$ and $f\left(x+\lambda_{1} / 4+\lambda_{2} / 4\right)$. The sensor output contains two harmonic components such as $A_{1} \sin \left(2 \pi x / \lambda_{1}+\alpha_{1}\right)+A_{1} \sin \left(2 \pi x / \lambda_{1}+\alpha_{2}\right)$, where $\alpha_{1}$ and $\alpha_{2}$ are phase angles. We can easily see the sum of all sensors becomes zero.

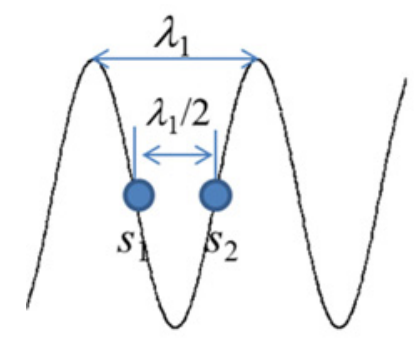

(a) Two sensors

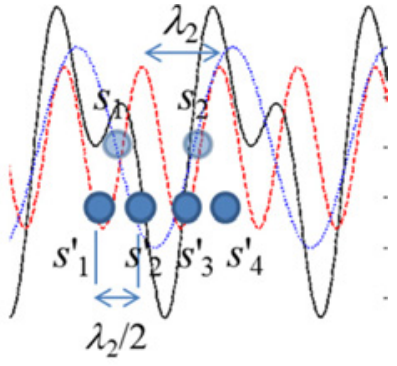

(b) Four sensors
Fig. 1 Extension of sensor array

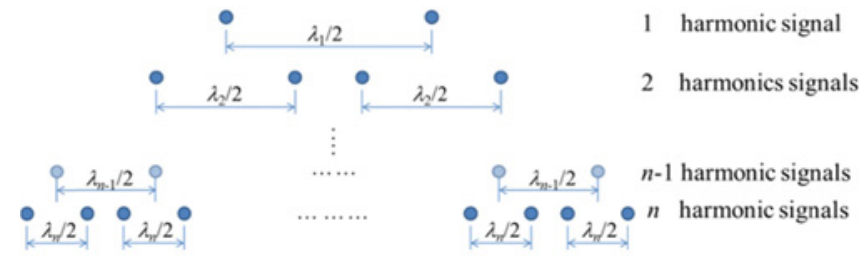

Fig. 2 1D sensor array for filtering out $n$ harmonic components
Now, we can extend and generalize this fact to a sensor array for filtering $n$ harmonic components $\left(\lambda_{1}>\lambda_{2}>\ldots>\lambda_{n}\right)$. At least, properly located $2^{n}$ sensors are necessary to filter out $n$ harmonic components. If the origin is the center of the sensor array, location of the $i^{\text {th }}$ sensor, $L_{i}$ can be expressed mathematically as Eq. (2). Here, $\left(i-1 \mid 2^{n-k}\right)$ is the quotient of division of $2^{n-k}$ by $i-1$.

$$
L_{i}=\sum_{k=1}^{n}-(-1)^{\left(i-1 \mid 2^{n-k}\right)} \frac{\lambda_{k}}{4}, i=1,2 \ldots 2^{\text {n }}
$$

The sensor location is graphically described in Fig. 2. The first row denotes a sensor array to filter a harmonic component of wavelength $\lambda_{1}$. The second row denotes a sensor array to filter out two harmonic components of wavelength $\lambda_{1}$ and $\lambda_{2}$. Finally, we can generalize a sensor array to filter out $n$ harmonic components from wavelength $\lambda_{1}$ to $\lambda_{n}$ shown in the last row of Fig. 2 .

The total width $W_{n}$ of the sensor array to filter out $n$ harmonic components is the sum of all half wavelengths of $n$ harmonic components, as shown in Eq. (3).

$$
W_{n}=\sum_{k=1}^{n} \frac{\lambda_{k}}{2}
$$

Two rows of 1D sensor array are required to identify the direction of the signal since the direction or the phase of the signal is important in some applications such as displacement sensor or encoder. Each row of 1D sensor array is separated each other in one fourth of wavelength of a desired harmonic component, which generates sine and cosine waves. In addition, these sine and cosine outputs are compatible to typical sine encoder for general motion controller. In addition, the height of the magnet matrix from the hall sensor array can be estimated from the magnitude of the magnetic flux density, which is calculated from root mean square of sine and cosine outputs of the hall sensor array.

\subsection{D Sensor Array}

1D sensor array can easily be extended into 2D sensor array for multidimensional measurement of a magnet matrix. Many combinations such as $\mathrm{L}$ and cross shapes are possible as shown in Fig. 3. In addition, $m^{2}$ sensors can be saved if $m$ rows of 1D array are used to filter out 1D harmonic component. We can reduce $4\left(2^{2}\right)$ sensors if we use one of sensor configurations in Fig. 3.

\section{2D Sensor Array for Measuring Position of the Magnet Matrix for a Planar Motor}

\subsection{A Planar Motor System with Iron Core}

Schematic and photo of a planar motor system are shown in Fig. 4.

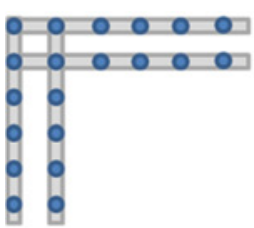

(a) L shape

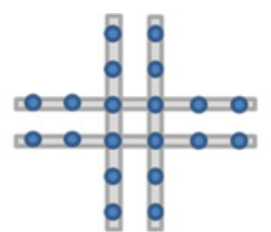

(b) Cross shape

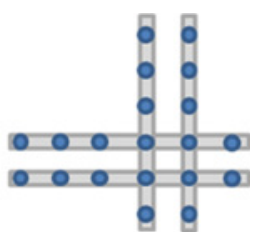

(c) General shape
Fig. 3 Various combinations of 1D sensor array 
The planar motor system consists of a mover with 2D magnet matrix, iron core array, a glass plate, air bearing pads, and a control system including sensors, amplifiers and motion controller. The specifications

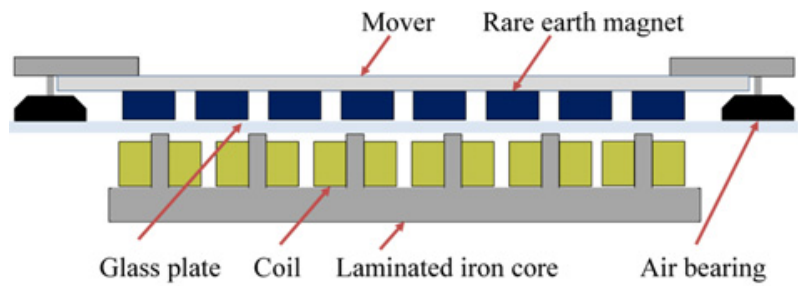

(a) Schematic

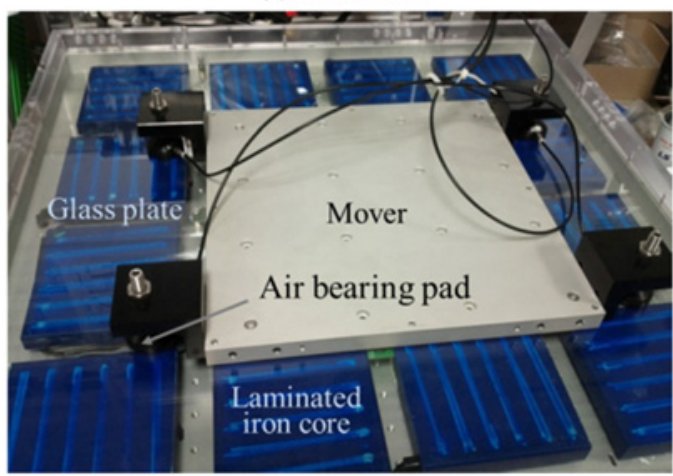

(b) Photo

Fig. 4 A planar motor system

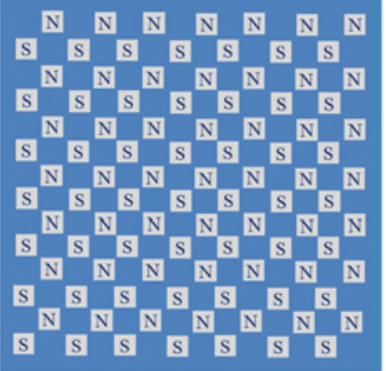

(a) Magnet matrix

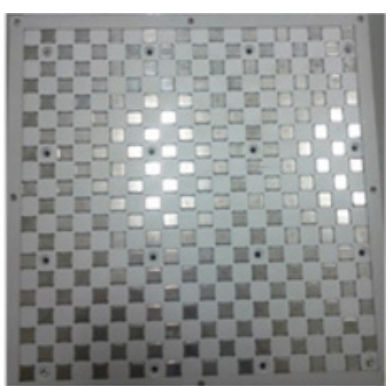

(b) Photo
Fig. 5 Mover with 2D magnet matrix ${ }^{20}$

Table 1 Specifications of the planar motor

\begin{tabular}{cc}
\hline Item & Specifications \\
\hline Stroke $\left(X \times Y \times \theta_{Z}\right)$ & $160 \mathrm{~mm} \times 160 \mathrm{~mm} \times \pm 5^{\circ}$ \\
\hline Maximum thrust force & $200 \mathrm{~N}$ \\
\hline $\begin{array}{c}\text { Capable load of air } \\
\text { bearing }\end{array}$ & $475 \mathrm{~N} /$ unit \\
\hline Mass of mover & $6.6 \mathrm{Kg}$ \\
\hline
\end{tabular}

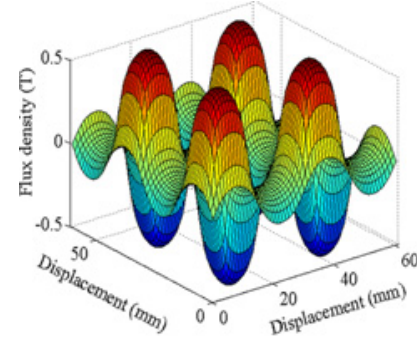

(a) Flux density (4mm height)

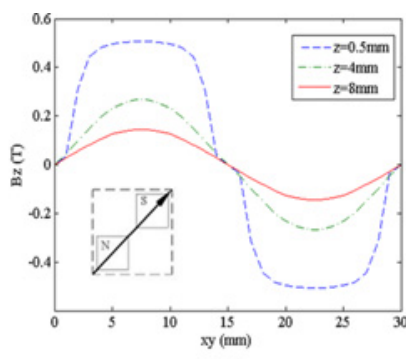

(b) Flux density according to height
Fig. 6 Magnetic flux density of 2D magnet matrix of the planar motor system are described in Table 1. The magnet matrix for the planar motor is not a Halbach array as shown in Fig. 5. Magnetic flux density distribution of the magnet matrix is calculated and shown in Fig. 6. The magnetic flux density is 2D sinusoidal and has various harmonics.

\subsection{D Hall Sensor Array for Measuring Displacement of the Magnet Matrix}

A 2D hall sensor array is designed both to decouple 2D sinusoidal magnetic flux density and to filter out harmonic components. Fundamental component should be removed to decouple the 2D magnetic flux density. In addition, most dominant second harmonic component is filtered simultaneously to reduce harmonic distortions.

2D hall sensor array with $\mathrm{L}$ shape is built to filter out first and second harmonic components, as shown in Fig. 7(a). 1D sensor array consists of 4 hall sensors that are separated in $1 / 8$ wavelength and filters out fundamental and second harmonics of the magnetic flux density. The first and second rows of the sensor array filter out fundamental and second harmonic of $\mathrm{X}$ directional magnetic flux density while they generate sine and cosine of $Y$ directional magnetic flux density. In the same manner, column array filters out $\mathrm{Y}$ directional magnetic flux density and generates $\mathrm{X}$ directional magnetic flux density.

A conditioning board for hall sensor array of Fig. 7(a) is shown in Fig. 7(b). The conditioning board consists of several stages of OP-amplifier circuits such as summation of hall sensor outputs and adjustment of gain and offset. The conditioned outputs of the 2D hall sensor array is fed into a motion controller and the motion controller calculates $\mathrm{X}$ and $\mathrm{Y}$ directional displacements using arctangent function. ${ }^{21,22}$ The motion controller adjusts offset ad then converts a relative position from arctangent function into the global position.

\subsection{Experiments}

Filtering performances of the 2D hall sensor array is experimentally verified using a XY linear motion stage in Fig. 8. The magnet matrix

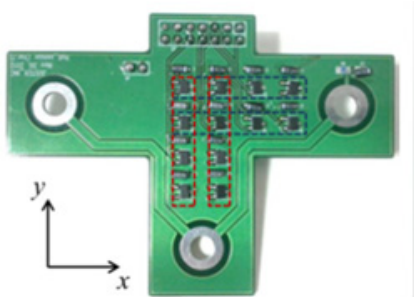

(a) $2 \mathrm{D}$ hall sensor array

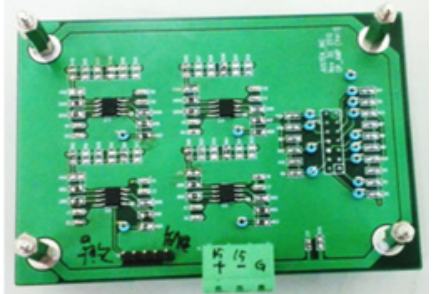

(b) Conditioning board
Fig. $72 \mathrm{D}$ hall sensor array and its conditioning board

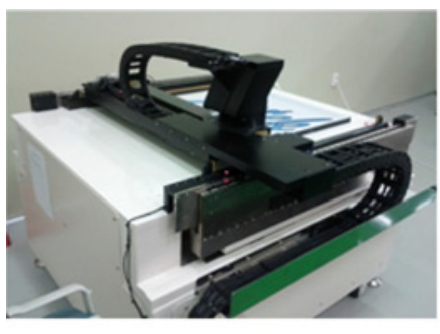

(a) XY linear stage

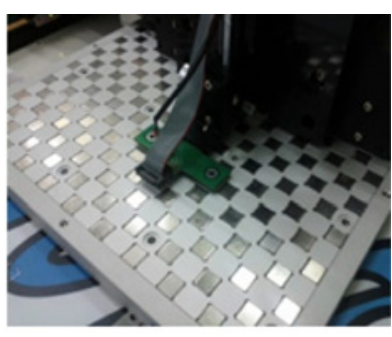

(b) Magnet and sensor array
Fig. 8 Experimental set-up for the hall sensor array 
is put on the base of the stage and the $2 \mathrm{D}$ hall sensor array is mounted on a manual $\mathrm{Z}$ stage that is attached to the mover head of the $\mathrm{XY}$ stage, as shown in Fig. 8(b). Then, conditioned signals are gathered at various heights and $\mathrm{XY}$ positions of the stage.

As the hall sensor array is mounted at 1, 3,5 and $8 \mathrm{~mm}$ height from the 2D magnet matrix, magnetic flux densities are measured and shown in Fig. 9. Fundamental and second harmonic components of magnetic flux density are filtered out. The conditioned output of the hall sensor array is saturated in case of $1 \mathrm{~mm}$ height and fourth harmonic component appears from $1 \mathrm{~mm}$ to $5 \mathrm{~mm}$ heights. As the result, almost pure sine wave magnetic flux density can be obtained at $8 \mathrm{~mm}$ height. If we want to measure the magnetic flux density at closer to the magnet matrix than $8 \mathrm{~mm}$, we need 1D array with 8 hall sensors to filter out fourth harmonic components. Magnitudes of harmonic components become strong as the $2 \mathrm{D}$ hall sensor array approaches the magnet matrix. The fourth harmonic components of magnetic flux can clearly be seen in Fig. 9(a) and (b), which cannot be filtered out with the 2D hall sensor array.

The four conditioned outputs of the hall at $8 \mathrm{~mm}$ height are shown in Fig. 10. The conditioning circuit generates sine and cosine signals of both $\mathrm{X}$ and $\mathrm{Y}$ directions from the 2D hall sensor array. There are small

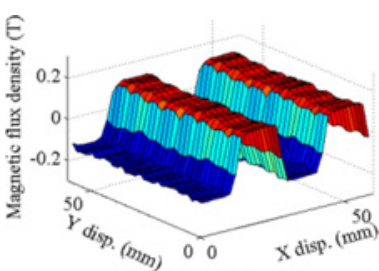

(a) $1 \mathrm{~mm}$ height

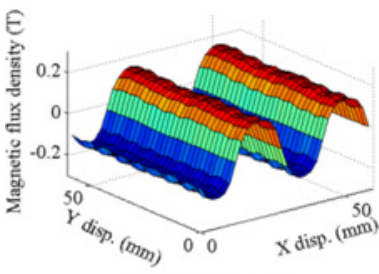

(c) $5 \mathrm{~mm}$ height

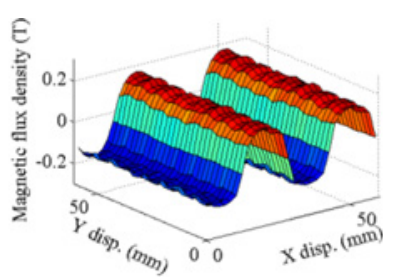

(b) $3 \mathrm{~mm}$ height

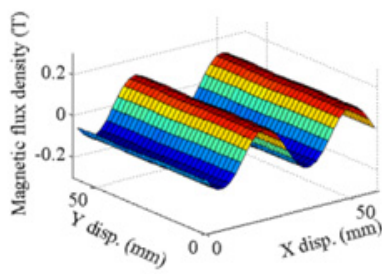

(d) $8 \mathrm{~mm}$ height
Fig. 9 Sensor output of the hall sensor array at variois heights

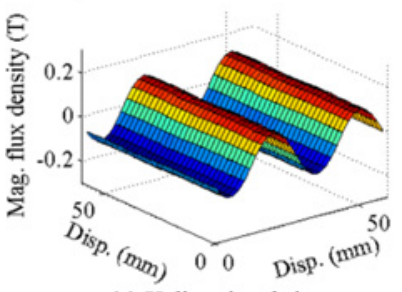

(a) $\mathrm{X}$ directional sine

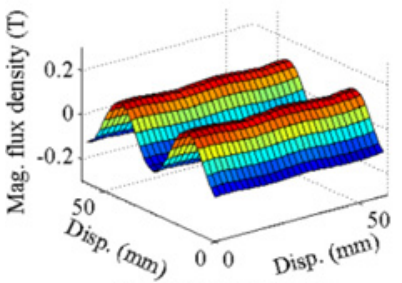

(c) $\mathrm{Y}$ directional sine

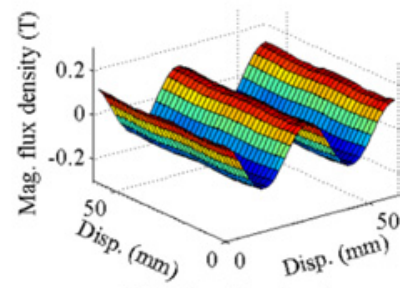

(b) $\mathrm{X}$ directional cosine

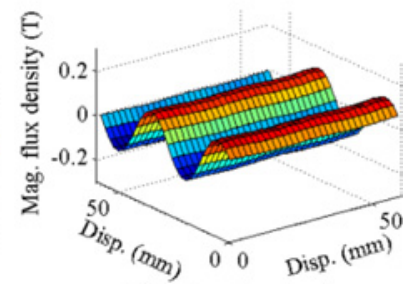

(d) Y directional cosine
Fig. 10 Sine and cosine outputs of conditioning circuit wave in magnitude of sine and cosine outputs, which are fundamental component of magnetic flux coming from the manufacturing tolerances and magnet or hall sensor irregularities.

$\mathrm{X}$ and $\mathrm{Y}$ displacements of the $2 \mathrm{D}$ magnet matrix are calculated and the displacement errors are evaluated in Fig. 11. Displacement is obtained using the phase angle from arctangent of the sine and cosine outputs of the conditioning board. ${ }^{21,22}$ The maximum error is about $1 \mathrm{~mm}$ and is too large to be acceptable. The errors consist of not only fundamental component of magnetic flux (magnet pitch), but also its multiples (small peaks between fundamental components). In particular, the fundamental component is intrinsic error caused by manufacturing tolerances and irregularity of hall sensors and magnets. These errors can be compensated with an error mapping technique. $^{14}$

The magnitude of each directional magnetic flux density is calculated and shown in Fig. 12(a) and (b). The magnitude of each directional flux density can be calculated using root mean square of sine and cosine outputs of conditioning circuit. Although the magnitude of each directional magnetic flux density is plat surface in ideal case, their magnitudes are not constant due to the manufacturing tolerances and harmonic components of the magnetic flux. In addition, average of each directional magnetic flux density is calculated and shown in Fig. 12(c), which can be used to estimate the height of the magnet matrix since magnitude of the magnetic flux density depends on the height of the magnet matrix. The average magnetic flux density looks like rectangular magnet matrix and reveals that there are still considerable harmonic components, which can be reduced by using Halbach magnet matrix or more hall sensors.

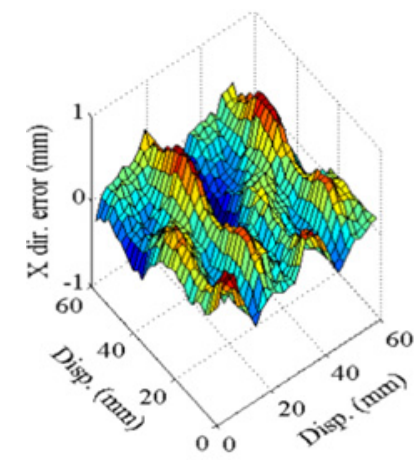

(a) $\mathrm{X}$ displacement

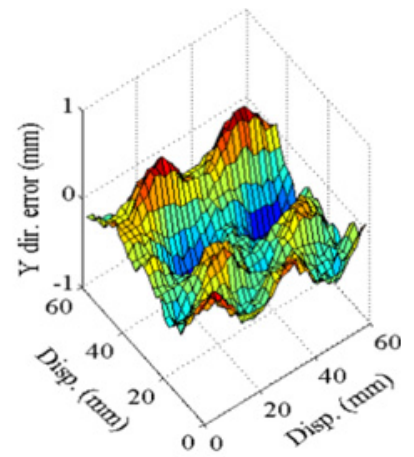

(b) Y displacement
Fig. 11 Calculated displacement errors of the hall sensor array

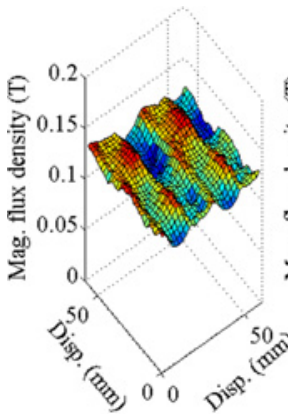

(a) $\mathrm{X}$ direction

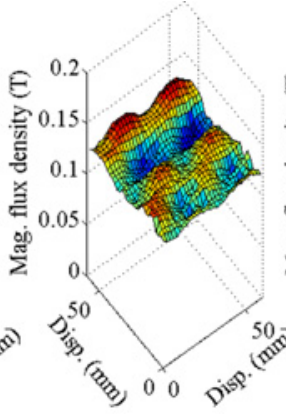

(b) $\mathrm{Y}$ direction

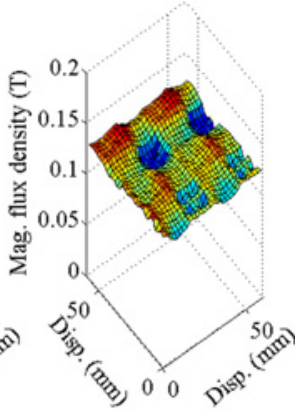

(c) Average
Fig. 12 Magnitude of directional magnetic flux density 


\section{Conclusions}

This paper presents a 2D hall sensor array to measure the position of a magnet matrix by filtering harmonic components of magnetic flux of the magnet matrix. A 1D sensor array is proposed to filter out arbitrary number of harmonic signals. Properly-located $2^{n}$ sensors are required to filter out $n$ harmonic components. In addition, the 1D sensor array is easily extended to a $2 \mathrm{D}$ sensor array. A 2D hall sensor array and its conditioning board are built to measure the position of the magnet matrix for a planar motor. The performance of the $2 \mathrm{D}$ hall sensor array is experimentally verified with a $\mathrm{XY}$ linear motor stage.

\section{ACKNOWLEDGEMENT}

This research was supported by the MSIP(Ministry of Science, ICT \& Future Planning), Korea, under the Convergence-ITRC support program (NIPA-2013-H0401-13-1004) supervised by the NIPA and by Basic Science Research Program through the National Research Foundation of Korea(NRF) funded by the Ministry of Education, Science and Technology (2013010764).

\section{REFERENCES}

1. Ouyang, P. R., Tjiptoprodjo, R. C., Zhang, W. J., and Yang, G. S., "Micro-Motion Devices Technology: The State of Arts Review," Int. J. Adv. Manuf. Technol., Vol. 38, No. 5-6, pp. 463-478, 2008.

2. Kim, W. J. and Trumper, D., "High-Precision Magnetic Levitation Stage for Photolithography," Precision Engineering, Vol. 22, No. 2, pp. 66-77, 1998

3. Cho, H. S., Im, C. H., and Jung, H. K., "Magnetic Field Analysis of 2-D Permanent Magnet Array for Planar Motor," IEEE Trans. Magn., Vol. 37, No. 5, pp. 3762-3766, 2001.

4. Compter, J., "Electro-Dynamic Planar Motor," Precision Engineering, Vol. 28, No. 2, pp. 171-180, 2003,

5. Kim, W. J., Bhat, N., and Hu, T., "Integrated Multidimensional Positioner for Precision Manufacturing,” J. Eng. Manuf., Vol. 218, No. 4, pp. 431-442, 2004.

6. Etxaniz, I., Izpizua, A., Martin, M. S., and Arana, J., "Magnetic Levitated 2D Fast Drive," IEEJ Transactions on Industry Applications, Vol. 126, No. 12, pp. 1678-1681, 2007.

7. Shinno, H., Yoshioka, H., and Taniguchi, K., “A Newly Developed Linear Motor-Driven Aerostatic X-Y Planar Motion Table System for Nano-Machining," CIRP Annals - Manuf. Tech., Vol. 56, No. 1, pp. 369-372, 2007.

8. Jansen, J. W., Van Lierop, C. M. M., Lomonova, E. A., and Vandenput, A. J. A., "Magnetically Levitated Planar Actuator with Moving Magnets," IEEE Transactions on Industry Applications, Vol. 44, No. 4, pp. 1108-1115, 2008.

9. Lu, X. and Usman, I. U. R., “6d Direct-Drive Technology for Planar
Motion Stages," CIRP Annals - Manuf. Tech., Vol. 61, No. 1, pp. 359-362, 2012.

10. Sawyer, B. A., "Magnetic Positioning Device," US Patent, No. 3457482 A, 1969.

11. Li, X., Gao, W., Muto, H., Shimizu, Y., Ito, S., and Dian, S., "A SixDegree-of-Freedom Surface Encoder for Precision Positioning of a Planar Motion Stage," Precision Engineering, Vol. 37, No. 3, pp. 771-781, 2013.

12. Li, X., Shimizu, Y., Ito, S., and Gao, W., "Fabrication of Scale Gratings for Surface Encoders by using Laser Interference Lithography with 405 nm Laser Diodes,” Int. J. Precis. Eng. Manuf., Vol. 14, No. 11, pp. 1979-1988, 2013.

13. Frissen, P. C. M., Compter, J. C., Peijnenburg, A. T. A., and Loopstra, E. R., “Displacement Device,” US Patent, No. 6879063, 2005.

14. Kawato, Y. and Kim, W. J., "Multi-Degree-of-Freedom Precision Position Sensing and Motion Control using Two-Axis Hall-Effect Sensors," Journal of Dynamic Systems, Measurement, and Control, Vol. 128, No. 4, pp. 980-988, 2006.

15. Lu, X., Rao, N., and Usman, I. U. R., "Six-Axis Position Measurement System for Levitated Motion Stages," CIRP Annals Manuf. Technol., Vol. 62, No. 1, pp. 507-510, 2013.

16. Halbach, K., "Design of Permanent Multipole Magnets with Oriented Rare Earth Cobalt Material," Nuclear Instruments and Methods, Vol. 169, No. 1, pp. 1-10, 1980.

17. Whitehouse, D. J., "Some Theoretical Aspects of Error Separation Techniques in Surface Metrology," Journal of Physics E: Scientific Instruments, Vol. 9, No. 7, pp. 531, 1976.

18. Ahn, H. J., Kim, I. H., and Han, D. C., "Nonlinear Analysis of Cylindrical Capacitive Sensor," Measurement Science and Technology, Vol. 16, No. 3, Paper No. 699, 2005.

19. Ahn, H. J. and Han, D. C., "Optimal Multi-Segment Cylindrical Capacitive Sensor," Measurement Science and Technology, Vol. 14, No. 5, Paper No. 531, 2003.

20. Binnard, M., "Planar Motor with Linear Coil Arrays," US Patent, No. 6445093 B1, 2002.

21. Honeywell, "HMR4007: Linear Position Sensor Module, 900289 01-03 Rev. A,” http://media.digikey.com/pdf/ Data \%20Sheets/ Honeywell\%20PDFs/HMR4007.pdf (Accessed 11 Mar. 2014)

22. Schott, C., Racz, R., Betschart, F., and Popovic, R. S., "Novel Magnetic Displacement Sensors," Paper No. SENSORS-003552002, 2002. 